\title{
First Report of Dieback Caused by Lasiodiplodia pseudotheobromae on Ormosia pinnata in China
}

\author{
Luoye Li, ${ }^{1}$ Mengying Lei, ${ }^{1}$ Honghong Wang, ${ }^{1}$ Xiaozhu Yang, ${ }^{1}$ Mebeaselassie Andargie, ${ }^{2, \dagger}$ and Shaobin Huang, ${ }^{1, \dagger}$ \\ ${ }^{1}$ Collaborative Innovation Center of Plant Pest Management and Bioenvironmental Health Application Technology, Guangdong \\ Eco-Engineering Polytechnic, Guangzhou 510520, China \\ ${ }^{2}$ Molecular Phytopathology and Mycotoxin Research, University of Göttingen, Göttingen, Germany
}

\begin{abstract}
Ormosia pinnata (Lour.) Merr. is an important tree used for landscape and plant recovery of barren slopes in China. During an investigation of plant disease on landscape trees in 2018, a dieback was observed on $O$. pinnata trees in Guangzhou, Guangdong Province, China. Symptoms were characterized by initial dryness of the twigs and eventual death of the whole branch of the tree. Isolations from symptomatic

dieback of $O$. pinnata. Based on morphological characteristics and molecular analysis of the internal transcribed spacer rDNA (ITS15.8S-ITS2) and partial sequence of the translation elongation factor $1 \alpha(\mathrm{EF} 1-\alpha)$, the fungus causing dieback on $O$. pinnata was identified as Lasiodiplodia pseudotheobromae. This is the first report of $L$. pseudotheobromae infecting $O$. pinnata in the world.
\end{abstract} branches yielded 13 isolates including two main morphotypes. Pathogenicity tests showed that isolate GDOP1 from Type I caused

Keywords: dieback, Lasiodiplodia pseudotheobromae, ITS, EF1- $\alpha$

The legume genus Ormosia Jacks. was established by G. Jackson (1811) and it comprises around 130 species, which are distributed in Southeast Asia, tropical America, and North Australia (Pennington et al. 2005). Ormosia pinnata (family Fabaceae) is an evergreen tree originating in Hainan, China. It is mainly distributed in Guangdong Province, Guangxi autonomous region, China, as well as Vietnam and Thailand. Because of its large crown and lush branches and leaves, it is often used as a landscape tree on streets and courtyards. Furthermore, it is often used as pioneer tree for vegetation restoration on the barren slopes of South China, since its root system can fix nitrogen, which improves soil fertility (Zhao et al. 2000).

During 2018, an investigation of plant disease on landscape trees was done in the four districts of Guangzhou (Tianhe, Baiyun, Huangpu, and Haizhu), Guangdong Province, China. A dieback on O. pinnata was observed for the first time in Tianhe district. Although few diseased trees were found, the symptoms were serious. Disease symptoms included black spots and necrosis on branches, branch withering, and eventual death of the diseased trees.

Lasiodiplodia pseudotheobromae (family Botryosphaeriaceae) is an important pathogen of a wide variety of plants covering a broad geographical distribution. It causes great loss to agriculture and forestry. For instance, it was reported to cause canker and shoot dieback on apple and resulted in the death of apple branches (Xue et al. 2019). It brought twig blight on blueberry and resulted

${ }^{\dagger}$ Corresponding authors: M. Andargie, mebhel@yahoo.com; and S. B. Huang, h3602@126.com

Funding: This research was supported by the characteristic innovation project funded by the Department of Education of Guangdong Province (grant number 2018GKTSCX006).

The author(s) declare no conflict of interest.

Accepted for publication 15 June 2020.

(c) 2020 The American Phytopathological Society in the death of diseased branches within 7 to 10 days (Wang et al. 2016). It is also responsible for dieback on English walnut (Li et al. 2016), branch blight of Bougainvillea spectabilis ( $\mathrm{Li}$ et al. 2015), and pedicel and peduncle discoloration of grapes (Dissanayake et al. 2015) in China. Furthermore, it caused cankers and dieback on Persian lime in Mexico (Bautista-Cruz et al. 2019), dieback of mango in Peru and Korea (Kwon et al. 2017; RodriguezGalvez et al. 2017), and trunk cankers in Acacia mangium in Venezuela (Castro-Medina 2014). In this paper, dieback on O. pinnata trees is reported for the first time. The objectives of the study were: (i) to isolate the causal pathogen and determine its virulence by pathogenicity tests on $O$. pinnata seedlings, and (ii) to identify the species of pathogens associated with dieback of $O$. pinnata using morphological and molecular approaches.

\section{Materials and Methods}

Plant sampling and fungal isolation. Ten symptomatic branches were collected from the diseased trees in Tianhe district of Guangzhou, Guangdong Province $\left(113.33^{\circ} \mathrm{E}, 23.17^{\circ} \mathrm{N}\right)$. A piece of diseased tissue from each branch was used to isolate pathogen. Each infected tissue sample was surface-sterilized by soaking in $75 \%$ ethanol solution for $45 \mathrm{~s}$, followed by three rinses in sterile distilled water, then dried on sterilized filter paper. The surface-sterilized diseased pieces were placed on potato dextrose agar (PDA) in Petri dishes and incubated at $25^{\circ} \mathrm{C}$ in the dark. To purify fungi, a hyphae tip was carefully picked from the edge of each colony using an elbow tweezer with a very small pointed tip under a dissecting microscope and transferred to new PDA for subculturing. Each isolate was purified at least twice by this method. The purified isolates were used for pathogenicity determination, morphological observation, and molecular identification.

Pathogenicity tests. Through artificial inoculation, pathogenicity tests were conducted on living seedlings of $O$. pinnata. From the two morphotypes, the representative isolates of each type (GDOPI and $G D O P 2$ ) were used to inoculate branches. Each fungal isolate was grown on PDA in the dark at $25^{\circ} \mathrm{C}$ for 5 to 7 days prior to inoculation. A mycelial plug inoculation method was used as reported previously by Kwon et al. (2017) and Bautista-Cruz et al. (2019). For wound inoculation, young healthy branches were surface 
disinfected by $75 \%$ alcohol and a small wound was made on each branch with a sterile knife. A piece of colonized PDA plug ( $8 \mathrm{~mm}$ in diameter) was placed into the wound and sealed with Parafilm (Bemis, U.S.A.) to keep moist until disease symptoms appeared. For inoculation without wounding, colonized PDA plugs were kept on unwounded branches and sealed with Parafilm. Branches of control seedlings were inoculated with PDA plugs without the pathogen. The plants were kept in a greenhouse for over 3 days until symptoms appeared and they were managed as normal. The experiments were conducted in 2018, 2019, and 2020. For each isolate or control, 30 seedlings of $O$. pinnata were used for pathogenicity tests in total.

Morphological analysis of the pathogen. Based on preliminary pathogenicity tests, the isolates of $G D O P 1$ and GDOP2 were inoculated on PDA and incubated at $25^{\circ} \mathrm{C}$ in the dark for 7 days. Colony characteristics on PDA including mycelium growth, color, and sporulation were observed. In addition, water agar media and autoclaved $O$. pinnata twigs were used to induce sporulation following the method of Alves et al. (2008). Briefly, fungal isolates were grown on autoclaved $O$. pinnata twigs on $2 \%$ water agar to observe sporulation. Conidiomata and sporulation on the twig were observed 7 days later.

Fungal structures such as conidia obtained from pycnidia of the symptomatic branches were observed with a Leica DM4B microscope to elucidate the conidial morphology. Photomicrographs were recorded and 50 conidia measurements were made (Leica DM4B or Leica DM750). Conidiomata on dead or dying branches were also observed with a dissecting microscope (Leica SPAO).

DNA extraction, PCR amplification and sequencing. The pathogen's DNA was extracted from mycelium cultured on PDA plates using GV-filamentous fungi genomic DNA extraction kit (Beijing Dingguo Changsheng Biotechnology Co. Ltd, Beijing, China) according to the manual. Target regions of the internal transcribed spacer (ITS) of the nuclear rDNA (ITS1-5.8S-ITS2) using ITS1 (5'TCCGTAGGTGAACCTGCGG-3') and ITS4 (5'-TCCTCCGCTTATT GATATGC-3') (White et al. 1990) primers were amplified using PCR. A $25 \mu \mathrm{l}$ PCR mixture was prepared, which contained $2.5 \mu \mathrm{l}$ of $10 \times \mathrm{PCR}$ buffer, $0.5 \mu \mathrm{l}$ of $10 \mu \mathrm{mol} /$ liter of each primer, $0.2 \mu \mathrm{l}$ of $5 \mathrm{U} / \mu \mathrm{l}$ ExTaq DNA Polymerase (Takara, China), $2 \mu$ l of dNTP mixture $(2.5 \mathrm{mmol} /$ liter of each), $1 \mu$ INA template, and $\mathrm{ddH}_{2} \mathrm{O}$ to $25 \mu$ l. The PCR conditions for amplification of the ITS-rDNA region were initial denaturation at $94^{\circ} \mathrm{C}$ for $4 \mathrm{~min} ; 30$ cycles of denaturation at $94^{\circ} \mathrm{C}$ for $30 \mathrm{~s}$, annealing at $58^{\circ} \mathrm{C}$ for $30 \mathrm{~s}$, and extension at $72^{\circ} \mathrm{C}$ for $45 \mathrm{~s}$; and final extension at $72^{\circ} \mathrm{C}$ for $7 \mathrm{~min}$.

The EF1- $\alpha$ was amplified and sequenced with primers EF1-688F (5'-CGGTCACTTGATCTACAAGTGC-3') and EF1-1251R (5'CCTCGAACTCACCAGTACCG-3') (Alves et al. 2008). The PCR mixture was the same as that of the ITS-rDNA, except that the primers were replaced by EF1- $\alpha$ primers. The PCR conditions for amplification of the EF1- $\alpha$ region were initial denaturation at $94^{\circ} \mathrm{C}$ for $4 \mathrm{~min} ; 30$ cycles of denaturation at $94^{\circ} \mathrm{C}$ for $30 \mathrm{~s}$, annealing at $56^{\circ} \mathrm{C}$ for $30 \mathrm{~s}$, and extension at $72^{\circ} \mathrm{C}$ for $1 \mathrm{~min}$; and final extension at $72^{\circ} \mathrm{C}$ for $7 \mathrm{~min}$. PCR products were sequenced at IGE Biotechnology LTD in Guangzhou, China. The newly obtained sequences were deposited in the GenBank database with the accession numbers MN294610 and MN326014.

Sequence alignment and phylogenetic analysis. The resulting ITS-rDNA and EF1- $\alpha$ sequences were compared with the GenBank database using BLASTN at the National Center for Biotechnology Information (NCBI, https://www.ncbi.nlm.nih.gov). Closely related and previously published sequences were selected for phylogenetic analyses. Analysis was done according to a previously published methodology by Wang et al. (2019). A phylogenetic tree was constructed using MrBayes 3.2. The MCMC (Markov Chain Monte Carlo) method was performed to estimate the posterior probabilities of the phylogenetic trees and generate a 50\% majority rule consensus tree. TREEVIEW1.6 was used to display and edit the trees. Sequences of $D$. seriata and $D$. mutila were used as outgroup.

\section{Results}

Fungal isolation. A total of 13 isolates were obtained from diseased tissue samples. Morphologically, there were mainly two morphotypes (Type I and Type II). Six isolates of Type I and five isolates of Type II were identified, which accounted for $85 \%$ of the total isolates. After 4 days on PDA, the mycelium of Type I was dense and became grayish black, but that of Type II was rare and white. Under artificial culture condition, only Type II had sporulation. One isolate of each type (named GDOP1 and GDOP2, respectively) was selected for the following study. The remaining two fungal isolates morphologically differed from each other, which might be from the air or other sources.

Pathogenicity tests. The isolates of GDOP1 and GDOP2 were selected to perform pathogenicity tests. Healthy branches were inoculated with both fungal isolates, GDOP1 and GDOP2, respectively.
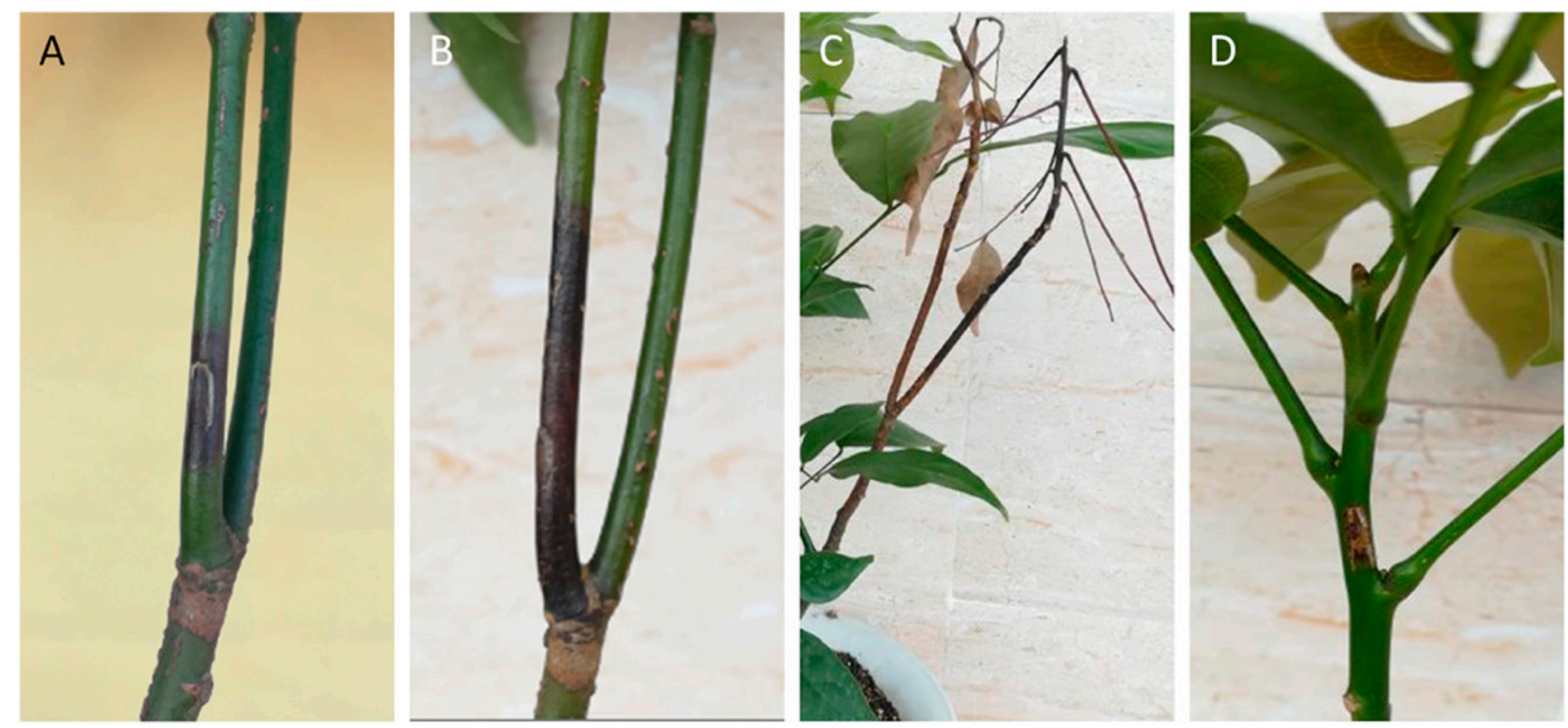

Fig. 1. Symptom of dieback disease on 0 . pinnata $A, 3$ days after inoculation with $G D O P 1 ; \mathbf{B}, 8$ days after inoculation with $G D O P 1$ isolate; $\mathbf{C}, 17$ days after inoculation with $G D O P 1$ isolate; and $\mathbf{D}, 17$ days after inoculation with GDOP2 isolate. 
At the third day after inoculation, the inoculation point of the wounded branch that was inoculated with GDOP1 became black (Fig. 1A). Later, the black spots expanded rapidly, covering the branches; even the leaves on these branches eventually dried (Fig. $1 \mathrm{~B}$ and C). Lastly, all the dried leaves fell and the branches themselves dried fully. The symptoms of these branches were consistent to those of diseased plants observed in the field. Even though the loci of the wounded branch inoculated with GDOP2 became dark, the dark spots did not expand, and dryness was observed only on the area where inoculation was performed, and the branches grew normally (Fig. 1D). No symptoms were observed on unwounded or control branches. Similar results were obtained during the whole study period. From the branches inoculated with GDOP1, we successfully reisolated the same isolate as the original one. Thus, it was confirmed that $G D O P 1$ was the causal agent of dieback of $O$. pinnata.

Morphological characteristics. The mycelia of GDOP1 grew very fast on PDA. Mycelia spread and covered the whole 9-cm Petri dish 3 days after inoculation. Aerial mycelia grew vigorously and occasionally grew up to the Petri dish cover. The mycelia were initially white. After 4 days of inoculation, the color deepened and eventually turned black (Fig. 2A). GDOP1 did not produce spores on PDA, water agar media, or on autoclaved $O$. pinnata twigs on water agar.

Many conidiomata were observed on the diseased branches that were infected by GDOP1. The conidiomata were semi-immersed, near spherical, and black. The top of conidiomata protruded out of the plant epidermis (Fig. 2B). Two kinds of conidia were observed in these conidiomata. One was an immature type, hyaline and aseptate (Fig. 2D). The other was a mature type, dark brown, 1-median septate, and had a longitudinally striate appearance (Fig. 2E). Conidia of $G D O P 1$ were ellipsoid, 17.8 to $28.6 \times 12$ to $16.7 \mu \mathrm{m}$ (avg. of 50 conidia $=23.5 \times 13.8 \mu \mathrm{m}, \mathrm{L} / \mathrm{W}$ ratio $=1.7$, range from 1.32 to
2.28). These morphological characteristics of GDOP1 corresponded to L. pseudotheobromae (Alves et al. 2008; Wang et al. 2016; Xue et al. 2019).

Molecular identification and phylogenetic analyses. The amplicon size of ITS and EF1- $\alpha$ sequence of GDOP1 were 474 and $534 \mathrm{bp}$ respectively. These two sequences were deposited in GenBank (accession nos. MN294610 and MN326014). NCBI BLASTn search showed that GDOP1 was most similar to L. pseudotheobromae. ITS sequence showed $99.79 \%$ similarity to that of published L. pseudotheobromae isolates CBS 116459 (type specimen of L. pseudotheobromae), CBS 116460, CBS 304.79, and CBS 374.54 while EF1- $\alpha$ sequence showed $99.67 \%$ similarity to that of CBS 116459, CBS 116460, and CBS 374.54 and $99.35 \%$ similarity to CBS 304.79 . In the phylogenetic tree based on the ITS and EF1- $\alpha$ sequences, the isolate GDOP1 clustered within a clade comprising reference isolates of $L$. pseudotheobromae (Fig. 3). Based on symptoms, pathogenicity, morphological characteristics, and molecular data, GDOP1 (causing dieback of $O$. pinnata) was identified as L. pseudotheobromae.

\section{Discussion}

Dieback was newly found on O. pinnata in Guangzhou, China. Although few infected plants were observed in our survey, attention should be given to this disease since it has severe symptoms and may kill $O$. pinnata trees. In this study, the pathogen causing dieback of $O$. pinnata was identified as L. pseudotheobromae based on symptoms, pathogenicity, morphological characteristics, and molecular data. To our knowledge, this is the first report of L. pseudotheobromae causing dieback on $O$. pinnata in the world.

Dieback on $O$. pinnata seedlings was observed on almost all wounded branches $(>90 \%)$ that were artificially inoculated by GDOP1. No symptoms were observed on unwounded branches in this study. These results showed that GDOP1 invades $O$. pinnata
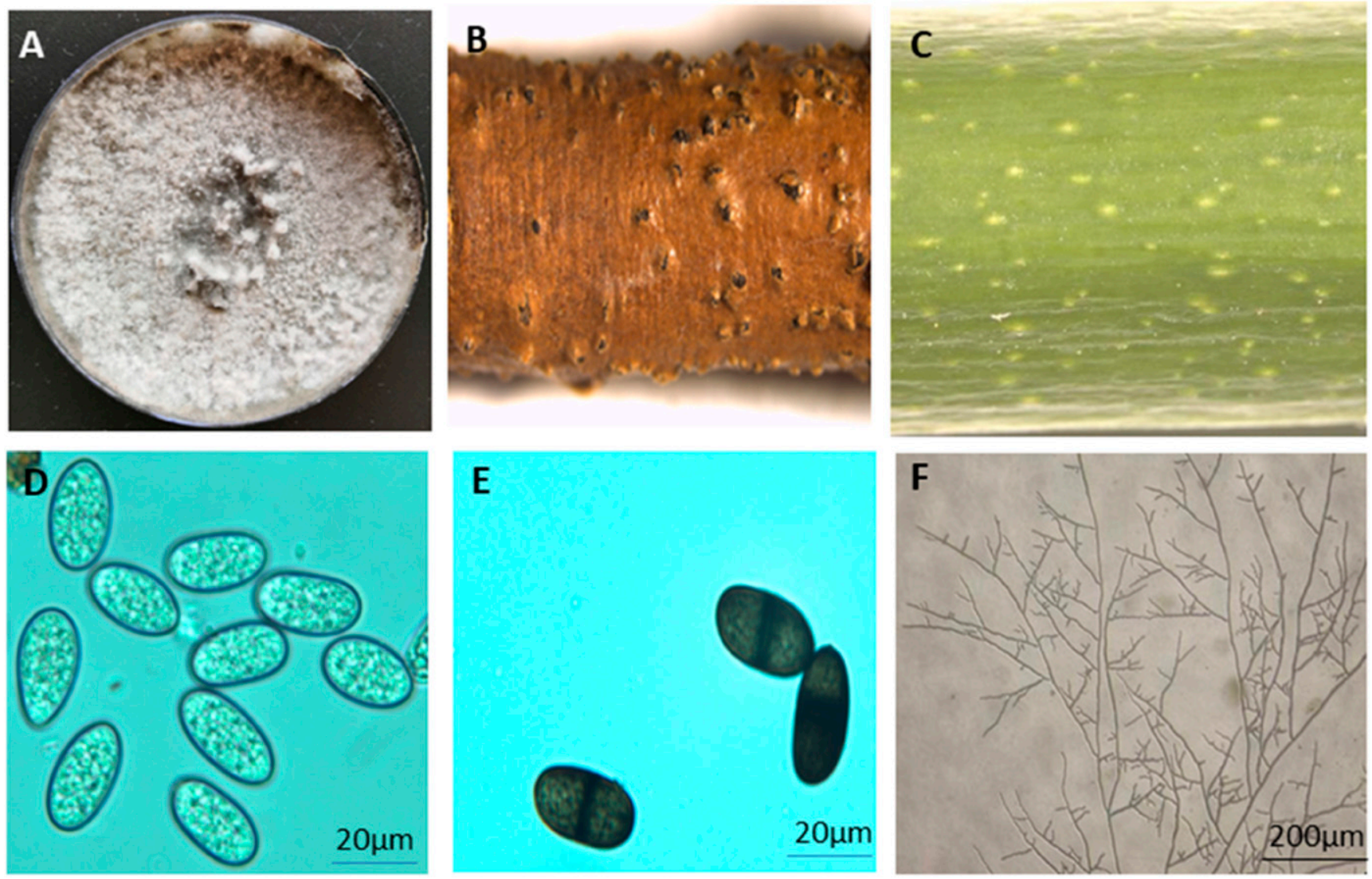

Fig. 2. Colony and conidial morphology of GDOP1. A, Colony morphology of a 15-day-old sample; B, conidiomata on diseased branches; C, uninfected healthy branch; D, immature conidia (whitish) with thin walls; E, mature conidia (dark brown) with septa and thick walls (scale bars: $20 \mu \mathrm{m}$ ); F, mycelia of GDOP1 (scale bar: $200 \mu \mathrm{m}$ ). 
trees mainly through wounds. This is consistent with previous studies (Xu et al. 2013).

L. pseudotheobromae was first described as a new species in 2008 (Alves et al. 2008). It is difficult to distinguish L. pseudotheobromae and L. theobromae just by their morphological characteristics. Therefore, DNA sequences of ITS and EF1- $\alpha$ genes were usually used to identify these species (Alves et al. 2008; Dou et al. 2017; Xue et al. 2019; Zhang et al. 2019). In this study, not only did the morphology of GDOP 1 correspond to L. pseudotheobromae, but also the sequences of ITS and EF1- $\alpha$ showed high homology to those of L. pseudotheobromae. Phylogenetic analyses also showed that GDOP1 was closest to L. pseudotheobromae. Therefore, the pathogen causing dieback on $O$. pinnata GDOP1 was determined as L. pseudotheobromae.

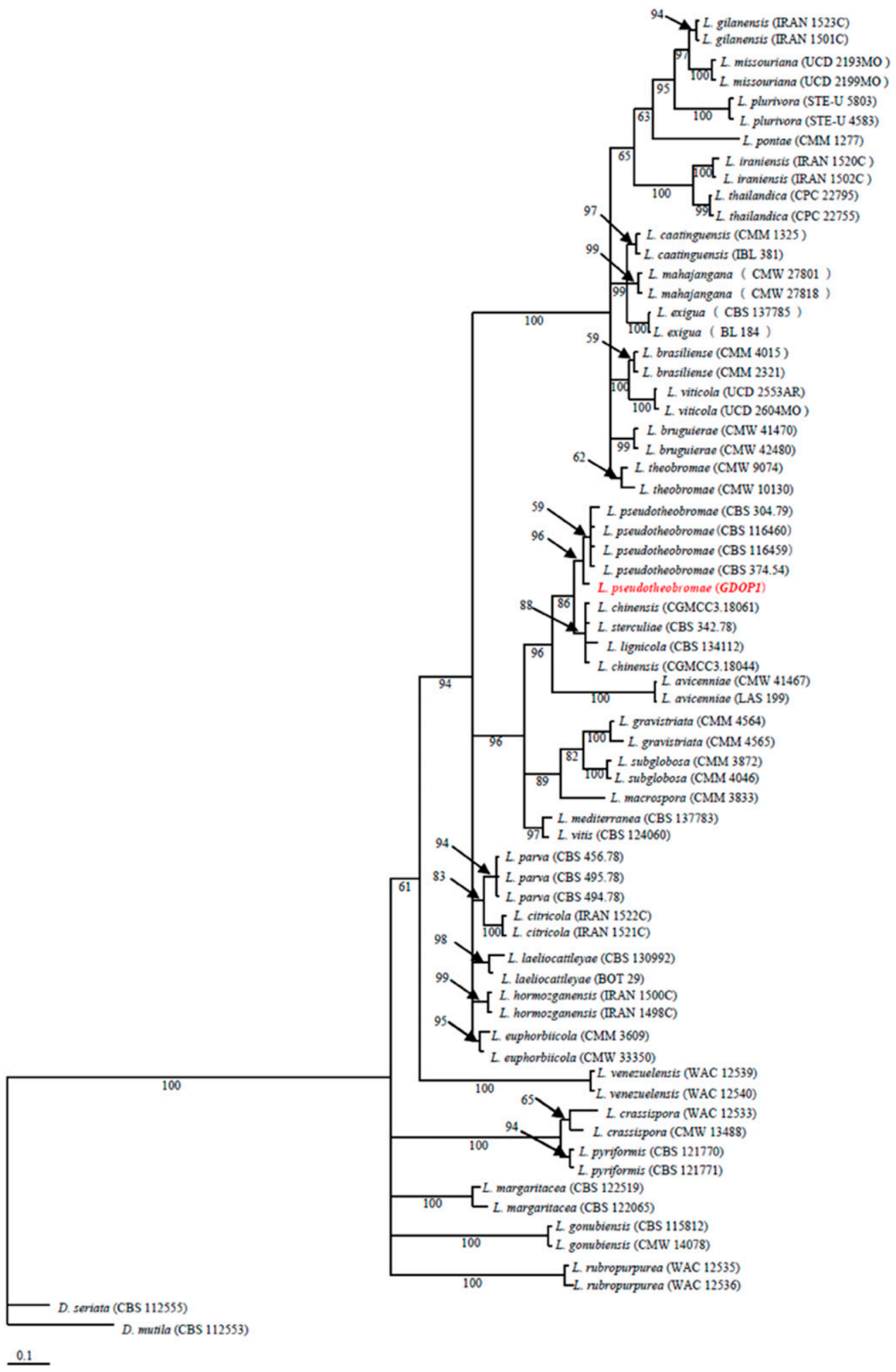

Fig. 3. Bayesian consensus tree generated from sequences of the combined ITS and TEF1- $\alpha$ dataset. Posterior probability values exceeding $50 \%$ are given on appropriate clades. Newly obtained sequences are indicated in bold. 


\section{Literature Cited}

Alves, A., Crous, P. W., Correia, A., and Phillips, A. J. L. 2008. Morphological and molecular data reveal cryptic speciation in Lasiodiplodia theobromae. Fungal Divers. 28:1-13.

Bautista-Cruz, M. A., Almaguer-Vargas, G., Leyva-Mir, S. G., Colinas-León, M. T., Correia, K. C., Camacho-Tapia, M., Robles-Yerena, L., Michereff, S. J., and Tovar-Pedraza, J. M. 2019. Phylogeny, distribution and pathogenicity of Lasiodiplodia species associated with cankers and dieback symptoms of Persian lime in Mexico. Plant Dis. 103:1156-1165.

Castro-Medina, F. 2014. First report of Lasiodiplodia pseudotheobromae causing trunk cankers in Acacia mangium in Venezuela. Plant Dis. 98:686.

Dissanayake, A. J., Zhang, W., Liu, M., Ekachai, C., Ji, Y. Y., Li, X. H., and Kevin, D. H. 2015. Lasiodiplodia pseudotheobromae causes pedicel and peduncle discolouration of grapes in China. Australas. Plant Dis. Notes 10:21.

Dou, Z. P., He, W., and Zhang, Y. 2017. Lasiodiplodia chinensis, a new holomorphic species from China. Mycosphere 8:521-532.

Kwon, J. H., Choi, O., Kang, B. S., Lee, Y. Y., Park, J. Y., Kang, D.-W., Han, I. Y., and Kim, J. W. 2017. Identification of Lasiodiplodia pseudotheobromae causing mango dieback in Korea. Can. J. Plant Pathol. 39:241-245.

Li, G. Q., Liu, F. F., Li, J. Q., and Chen, S. F. 2016. Characterization of Botryosphaeria dothidea and Lasiodiplodia pseudotheobromae from English walnut in China. J. Phytopathol. 164:348-353.

Li, G. Q., Arnold, R. J., Liu, F. F., Li, J. Q., and Chen, S. F. 2015. Identification and pathogenicity of Lasiodiplodia species from Eucalyptus urophylla 3 grandis, Polyscias balfouriana and Bougainvillea spectabilis in Southern China. J. Phytopathol. 163:956-967.

Pennington, R. T., Stirton, C. H., and Schrire, B. 2005. Tribe: Sophoreae. Pages 227-251 in: Legumes of the World. G. Lewis, B. Schrire, B. Mackinder, and M. Lock, eds. Royal Botanic Garden, Kew, England.
Rodríguez-Gálvez, E., Guerrero, P., Barradas, C., Crous, P. W., and Alves, A. 2017. Phylogeny and pathogenicity of Lasiodiplodia species associated with dieback of mango in Peru. Fungal Biol. 121:452-465.

Wang, H. H., Zhuo, K., Cai, R. D., and Liao, J. L. 2019. Morphological and molecular characterisation of Paralongidorus sacchari (Nematoda: Longidoridae), a new record of needle nematode in China. Eur. J. Plant Pathol. 155:615-625.

Wang, J. N., Zhao, H. H., Yu, Y. Y., Li, X. D., Liang, Ch., and Li, B. D. 2016. The pathogen causing Lasiodiplodia twig blight of blueberry. Mycosustema. 35: 657-665 (in Chinese).

White, T. J., Bruns, T., Lee, S., and Taylor, J. 1990. Amplification and direct sequencing of fungal ribosomal RNA genes for phylogenetics. Pages 315-322 in: PCR Protocols: a guide to methods and applications. M. A. Innis, D. H. Gelfand, J. J. Sninsky, and T. J. White, eds. Academic Press, New York.

Xu, C. N., Zhou, Z. S., Chi, F. M., Wu, Y. X., Ji, Z. R., and Zhang, H. J. 2013. Research on the pathogen causing Botryosphaeria stem blight on blueberry. Acta Hortic. Sin. 40:31-236 (in Chinese).

Xue, D. S., Meng, L. L., Li, G. F., Li, B. H., and Wang, C. X. 2019. First report of Lasiodiplodia pseudotheobromae causing canker and shoot dieback on apple in China. Plant Dis. 103:2478.

Zhang, Q., Hao, Y. Y., Wu, X. Y., Xu, J. J., Long, H., Zhang, Y., and Wang, Y. 2019. A new disease of Philodendron selloum caused by Lasiodiplodia theobromae. Acta Phytopathol. Sinica 49:276-280 (in Chinese).

Zhao, P., Zeng, X. P., Peng, S. L., and Sun, G. C. 2000. Daily variation of gas exchange, stomatal conductance and water use efficiency in summer leaves of Ormosia pinnata. J. Trop. Subtrop. Bot. 8:35-42 (in Chinese). 